-Vision-

\title{
A New Age of Secondary Batteries
}

Yoshiharu MATSUDA

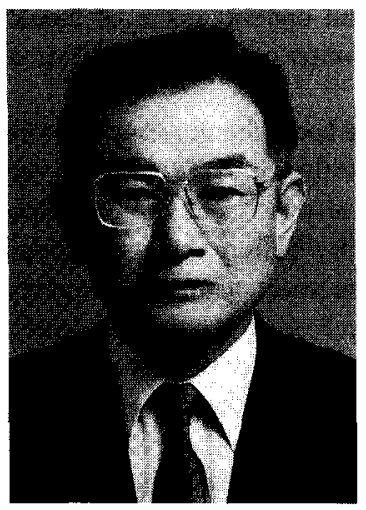

Battery technology and industry have extensively progressed for the last decade. In Japan, the output of nickel-cadmium secondary batteries has exceeded that of lead-acid secondary batteries for automobiles. The electronics industry has expanded tremendously. Such new electronic devices as cordless telephones, word processors, video cameras, personal computers and electronic toys have demanded a large amount of small-size secondary batteries. In fact, the consumption of high power density secondary batteries such as small-size nickel-cadmium secondary batteries have recently increased depending on the situation cited above.

On the other hand, lead-acid and nickel-cadmium batteries are now shunned from the viewpoint of environmental pollution by the harmful elements of lead and cadmium. Therefore, the development of new high power-density rechargeable batteries is expected. At the present, some kinds of small cylinder-type nickel - hydrogen and lithium ion secondary batteries are applied to the use in the same fields that have employed common secondary batteries though the consumption of those new batteries is limited.

For the development of new secondary batteries, it is essential to exploit advanced materials. Many kinds of advanced materials should be used as the anodes, cathodes, electrolytes, diaphragms (separators), current collectors, sealants, containers and so on. New hydrogen storage alloys have been used in nickel-hydrogen secondary batteries. Lithium (or lithium alloys, lithium inserting carbon), organic electrolytes, layered structure oxides and pore controlled polymer diaphragms have newly been applied to lithium secondary batteries.

The future market of new secondary batteries will expand wider and wider. The new category of secondary batteries includes micro batteries, paper batteries contained in intelligent cards, small high power-density batteries used in electronic devices, high power- and high energy-density batteries for the energy source of electric vehicles, and stationary batteries for electrical load levelling. However, high reliability, safety and nonpollution are indispensable as well as economy for the new batteries.

Newer batteries consisting of advanced materials are now under development and are still expected. A new age of secondary batteries is coming.

Chairman, Battery Technology Committee of the Electrochemical Society of Japan. Department of Applied Chemistry and Chemical Engineering, Yamaguchi University (Tokiwadai, Ube 755, Japan) 